\title{
Eventos pluviais intensos e seus impactos em Campina Grande-PB
}

\author{
Intense rainfall events and their impacts in Campina Grande-PB \\ SENA $^{1}$, J. P. O.; LUCENA ${ }^{2}$, D. B.; MORAES NETO ${ }^{3}$, J. M. \\ jariceliasena@hotmail.com
}

\section{Resumo}

A pluviometria é um fator importante para a caracterização do clima de uma determinada região. Portanto, o conhecimento da quantidade, intensidade e frequência da pluviosidade é de extrema importância para o desenvolvimento socioambiental de uma localidade. Nesse trabalho objetivou-se analisar a frequência de chuvas diárias, iguais ou superiores a $50 \mathrm{~mm} / \mathrm{dia}$, e seus impactos no município de Campina Grande - PB, no período de janeiro de 1994 a julho de 2018. Os dados de chuvas diárias foram obtidos na Agência Executiva de Gestão das Águas do Estado da Paraíba (AESA) para o posto Campina Grande/Embrapa. Foram identificados 30 eventos de chuvas diárias com intensidade acima de $50 \mathrm{~mm} / \mathrm{dia}$, com maior frequência de registro, no ano de 2011,7 eventos extremos. Em escala mensal, os eventos extremos encontram-se dentro do período chuvoso do município(maio a julho). No ano de 2018 foi registrado em 10 de fevereiro um evento com um volume pluviométrico de $56,8 \mathrm{~mm}$, com duração de menos que $2 \mathrm{hs}$, que segundo a Defesa Civil causou vários prejuízos à sociedade de Campina Grande - PB.

Palavras-Chave: Pluviosidade, escala diária, eventos extremos.

\begin{abstract}
Pluviosidad is an important factor for characterizing the climate of a given region. Therefore, knowledge of the quantity, intensity and frequency of pluviosidad is of extreme importance for the social and environmental development of a locality. The objective of this study was to analyze the frequency of daily rainfall, equal to or greater than $50 \mathrm{~mm} /$ day, and its impacts in the city of Campina Grande - PB, from January 1994 to July 2018. Daily rainfall data were obtained from the Paraíba State Water Management Agency (AESA) for Campina Grande / Embrapa. We identified 30 daily rainfall events with intensity above $50 \mathrm{~mm} /$ day, with the highest frequency of recording in 2011, 7 extreme events. On a monthly scale, extreme events are within the municipality's rainy season (May to July). In the year 2018, an event with a rainfall of $56.8 \mathrm{~mm}$ was recorded on February 10, lasting less than 2 hours, which according to the Civil Defense caused several losses to the company of Campina Grande - PB.
\end{abstract}

Keywords: Pluviosidad, daily scale, extreme events.

\section{INTRODUÇÃO}

A precipitação pluvial é importante para a caracterização do clima de uma determinada região, interferindo sobre vários aspectos e atividades relacionadas a sociedade (agricultura, pecuária, etc). O excesso (enchentes) ou escassez (secas) de chuvas causam danos socioeconômicos e ambientais de grande dimensão (DINIZ, 2013).

\footnotetext{
${ }^{1}$ Jaricélia Patrícia de Oliveira Sena, Programa de Pós-Graduação em Engenharia Agrícola, Universidade Federal de campina Grande, Campina Grande-PB, Brasil.

${ }^{2}$ Daisy Beserra Lucena, Departamento de Geociências, Universidade Federal da Paraíba, João Pessoa-PB, Brasil.

${ }^{3}$ João Miguel Moraes Neto, Departamento de Engenharia Agrícola, Universidade Federal de Campina Grande, Campina Grande-PB, Brasil.
} 
Segundo Vianello (1991) um importante instrumento que contribui para um melhor prognóstico desses eventos extremos (excesso ou escassez) de chuva, é o monitoramento diário do regime pluviométrico sobre uma dada localidade.

O relatório do Painel Intergovernamental sobre Mudanças Climáticas (IPCC) de 2012 aborda os eventos extremos como sendo àqueles eventos climáticos e meteorológicos acumulados que ocasionam impactos, econômicos, sociais e ambientais. Segundo Marengo (2009) os eventos extremos acontecem quando valores, frequência e associação temporal das observações registram um aumento ou uma diminuição expressiva durante um determinado estado climático.

Os eventos extremos são responsáveis pela segunda maior frequência de desastres naturais no Brasil (CEPED/UFSC, 2012; BRASIL, 2014). Esses desastres podem ser de ordem hidrológica, como por exemplos, enxurradas e inundações bruscas e graduais, de natureza geológica, como deslizamento e erosão e de natureza meteorológica, as chuvas intensas (TEMOTEO et al, 2016).

Uma das possíveis causas dos danos deflagrados por episódios de chuva extrema tem sido o processo de urbanização desordenado presente nas grandes cidades brasileiras, as quais atingem, principalmente, as populações mais vulneráveis (LOUREIRO, 2014). Portanto, o conhecimento da quantidade, intensidade e frequência da pluviosidade é de extrema importância para o desenvolvimento socioambiental de uma localidade.

Segundo Minuzzi e Sediyama (2004) chuvas diárias maior que $50 \mathrm{~mm} / \mathrm{dia}$ é classificada como uma chuva extrema. É importante destacar que, alguns autores como, Gonçalves (2003), Zanelle, Sales e Abreu (2009) e Silva (2014) consideram um limiar de 60 $\mathrm{mm} /$ dia como chuva extrema.

Nesse contexto, objetivou-se analisar a frequência de chuvas diárias, iguais ou superiores a $50 \mathrm{~mm} / \mathrm{dia}$, e seus impactos no município de Campina Grande - PB, no período de janeiro de 1994 a julho de 2018.

\section{METODOLOGIA}

O município de Campina Grande está localizado na mesorregião do agreste paraibano, na porção oriental do Planalto da Borborema, região semiárida do nordeste brasileiro. Segundo a classificação de Köppen o tipo de clima encontrado é As' (quente e úmido com chuva de outono-inverno), a temperatura média do ar é de $23,3^{\circ} \mathrm{C}$, com mínima de $17,7^{\circ} \mathrm{C}$ 
registrada no mês de julho e máxima de $30,1^{\circ} \mathrm{C}$ no mês de janeiro (SANTOS, 2011; SILVA, ALMEIDA, 2012).

Figura 1: Localização do município de Campina Grande-PB.

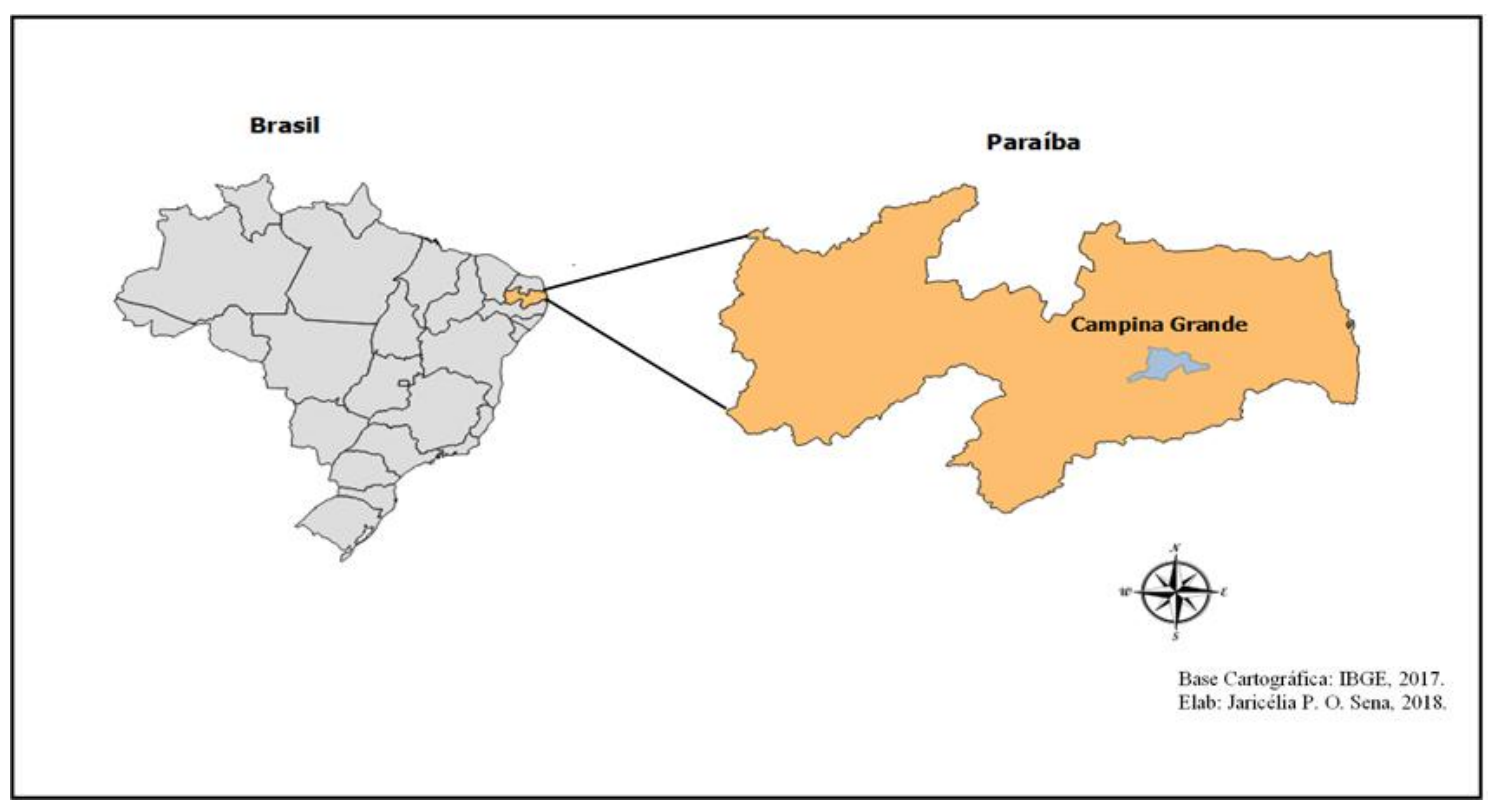

A agricultura do município constitui uma atividade de grande relevância na economia, é dependente totalmente das chuvas, já que a maioria delas são de sequeiro, ou seja, o agricultor depende unicamente do regime local de chuvas. Os principais produtos agrícolas são: algodão herbáceo, feijão, mandioca, milho, sisal, arroz, batata doce e inglesa, cebola, fava, fumo, mamona, melão, tomate e diversos produtos hortifrutigranjeiros (SANTOS, 2011).

Para a identificação dos eventos de chuvas extremos foram utilizados os dados diários de precipitação pluvial, obtidos da Agência Executiva de Gestão das Águas doEstado da Paraíba (AESA) para o posto Campina Grande/Embrapa, com localização geográfica na latitude sul de $7^{\circ} 22^{\prime} 56^{\prime}$ " e longitude oeste de 35 90' 42”, do período de janeiro de 1994 a julho de 2018. Os dados foram organizados no software Microsoft Office Excel 2013, para identificação e frequência (absoluta e relativa) dos eventos acima de $50 \mathrm{~mm} /$ dia.

A classificação dos totais anuais de chuva com relação a média em anos habitual, seco e chuvoso foi feito com base no cálculo do desvio padrão da série. Em que, precipitações pluviais acima da média mais o desvio padrão tem-se anos chuvosos, valores abaixo da média menos o desvio padrão anos secos e entre os desvios padrão (positivo e negativo) tem-se os anos normais ou habituais (a variabilidade esperada). 


\section{RESULTADOS E DISCUSSÃO}

Na análise da precipitação pluviometrica, no período de 1994 a julho 2018, registrouse 30 eventos de chuvas diárias com intensidade acima de $50 \mathrm{~mm} /$ dia (Tabela 1).

Na década de 2000 registraram-se 12 eventos extremos; entre os anos de 2010 a 2018, contabilizaram-se 16 eventos extremos, destacando o ano de 2011, com 7 eventos extremos, o mais chuvoso da série, com total pluviométrico anual de 1494,4 mm, apresentando um aumento de $89 \%$ em relação a média climatológica.

Analisando a relação dos anos classificados como seco, chuvosos ou habituais com a ocorrência de eventos extremos, percebe-se que nos anos habituais existe uma pequena propensão a ocorrência de eventos, dos 19 anos que foram classificados nesta classe $42 \%$ não apresentou nenhum, os demais 1 ou 2 eventos.

Nos anos secos, que só foram dois, um apresentou evento extremo e outro não, sendo muito insuficiente para tentar analisar alguma relação. Nos anos chuvosos, parece uma relação mais clara, pois foram observados em todos os anos eventos extremos. Entretanto, cabe ressaltar que, os eventos extremos que ocorrem na escala diária, podem ser eventos isolados o que não tem muita relação com os totais acumulados anuais, a não ser que ocorressem com uma maior frequência (Tabela 1).

Tabela 1: Total pluviometrico anual e número de eventos de chuva acima de $50 \mathrm{~mm} / \mathrm{dia}$ em Campina GrandePB, para o período de 1994 a julho de 2018. Fonte: AESA, 2018. Organização: Autores, 2018.

\begin{tabular}{cccccc}
\hline & $\begin{array}{c}\text { TOTAL } \\
\text { PLUVIOMÉTRICO } \\
\text { ANUAL }\end{array}$ & $\begin{array}{c}\mathbf{N}^{\mathbf{D}} \text { DE } \\
\text { EVENTOS } \\
\text { EXTREMOS }\end{array}$ & $\begin{array}{c}\text { ANOS } \\
\text { ANOS }\end{array}$ & $\begin{array}{c}\text { TOTAL } \\
\text { PLUVIOMÉTRICO } \\
\text { ANUAL }\end{array}$ & $\begin{array}{c}\mathbf{N}^{\mathbf{0}} \text { DE } \\
\text { EVENTOS } \\
\text { EXTREMOS }\end{array}$ \\
\hline 1994 & 1040,5 & 0 & 2006 & 735,1 & 2 \\
1995 & 702,7 & 0 & 2007 & 754,3 & 0 \\
1996 & 817 & 1 & 2008 & 919,5 & 2 \\
1997 & 681,4 & 0 & 2009 & 1066,2 & 1 \\
1998 & 360,8 & 0 & 2010 & 699,1 & 2 \\
1999 & 503,7 & 1 & 2011 & 1494,4 & 7 \\
2000 & 1365,1 & 2 & 2012 & 604,5 & 2 \\
2001 & 743,5 & 2 & 2013 & 752,8 & 0 \\
2002 & 770,6 & 0 & 2014 & 705,5 & 1 \\
2003 & 613,6 & 0 & 2015 & 595,5 & 1 \\
2004 & 1228,6 & 3 & 2016 & 544,3 & 1 \\
2005 & 866,5 & 0 & 2017 & 607 & 1 \\
& & & $2018 *$ & 631,2 & 1 \\
\hline
\end{tabular}

(*) ano com série incompleta.

As células de cor cinza refere-se a um ano habitual (normal), na cor amarela um ano seco e na cor verde um ano chuvoso. 
Na Figura 2 estão representados todos os 30 eventos extremos de chuva, observa-se que 16 eventos ( $53 \%)$ apresentam-se entre 50 e $60 \mathrm{~mm}$ diários. Os eventos acima de 80 $\mathrm{mm} /$ dia são mais raros, apenas $\sim 17 \%$ sendo 1 no ano de 2001 e 4 no ano de 2011, que como supracitado, este último foi o ano que mais apresentou eventos extremos. Sobre este ano, 2011, segundo as informações do Boletim Climanálise (INMET, 2011), os principais sistemas que contribuiram para a ocorrência de chuvas acima da média em algumas áreas no leste da Região Nordeste foi o Distúrbio Ondulatórios de Leste (DOL) associado ao efeito de brisa marítima, que transportou muita umidade para o continente.

Figura 2: Os maiores registros acumulados de precipitação pluviométrica diária em Campina Grande durante o período de 1994 a julho 2018.

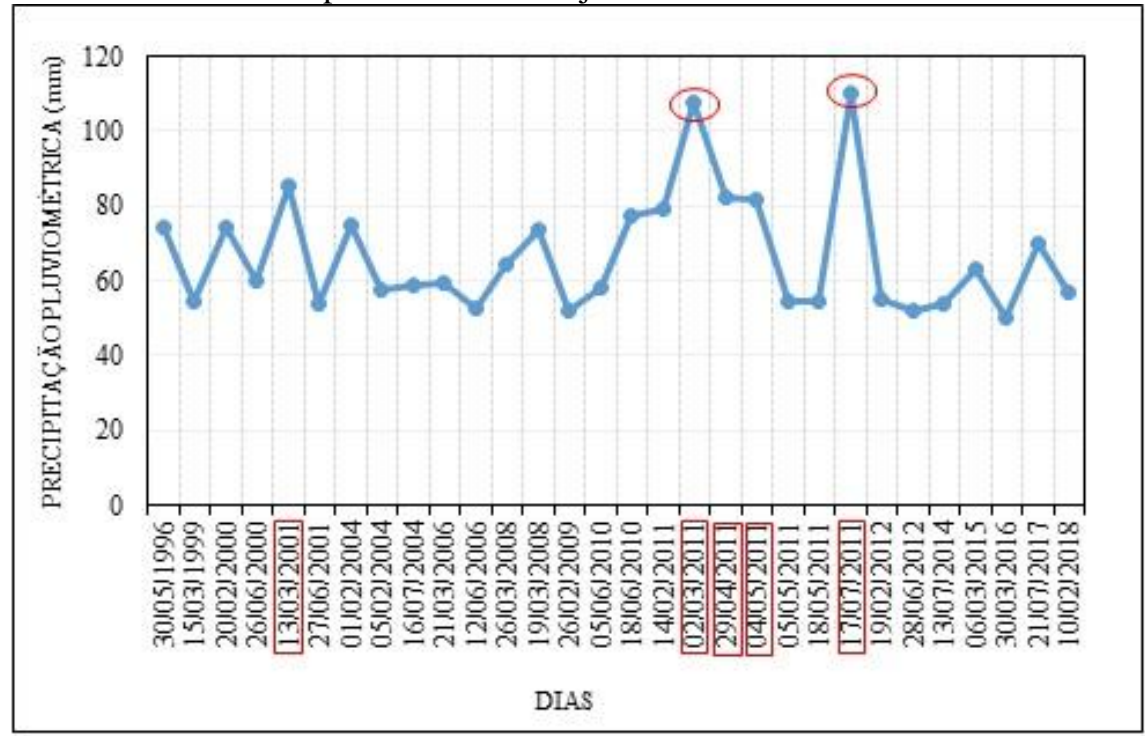

Os maiores eventos de excepcionalidade da série, foi no dia 02 de março de 2011, acumulando um total de 107,4 mm e no dia 17 de julho de 2011 um total de 110,7 mm (Figura 2). Em relação aos impactos destes eventos no município de Campina Grande, verifica-se que foi registrado o maior número de desastres naturais por inundações bruscas, somente no dia 17 de julho de 2011 foram quatro ocorrências, segundo o Centro Universitário de Estudos e Pesquisas sobre Desastres da Universidade Federal de Santa Catarina (CEPED/UFSC, 2012).

O Jornal eletrônico MaisPB noticiou que de acordo com a Defesa Civil 42 cidades da Paraíba no ano de 2011 foram atingidas pela chuva, dessas 30 com situação de emergência decretada pelo Governo do Estado, entre essas a cidade de Campina Grande. As fortes chuvas afetaram principalmente as pessoas (18.312 ocorrências registradas), além dos danos humanos 
causados. Também prejudicou o tráfego em 2.331 estradas gerando interdições das rodovias estaduais PB.

Analisando a frequência de eventos em escala mensal, observa-se que os meses de fevereiro a julho são os meses que registram mais eventos extremos, sendo março o mês de maior registro de ocorrências, com oito eventos (Tabela 2).

Desta forma, percebe-se que os registros de eventos extremos de chuva encontram-se dentro do período chuvoso do município, que compreende os meses de maio a julho segundo Silva (2014) e Temoteo (2016). Pode-se verificar também que nos meses considerados secos, fora da estação chuvosa, agosto-novembro, não tem registro de eventos extremos (Tabela 2).

Tabela 2: Frequência mensal dos eventos extremos de chuvas acima de 50 mm/dia em Campina Grande para o período de 1994 a julho 2018. Fonte: Autores, 2018.

\begin{tabular}{cccccccccccc}
\hline \multicolumn{11}{c}{ MESES } \\
\hline JAN & FEV & MAR & ABR & MAI & JUN & JUL & AGOS & SET & OUT & NOV & DEZ \\
0 & 7 & 8 & 1 & 4 & 6 & 4 & 0 & 0 & 0 & 0 & 0 \\
\hline
\end{tabular}

Analisando mês a mês do ano corrente, 2018, até o mês de julho, observou nos meses de janeiro, fevereiro, abril e maio pluviosidade acima da média esperada e nos meses de março, junho e julho uma diminuição das chuvas em relação a média (Figura 3).

Figura 3: Acumulado da precipitação pluviométrica em 2018 e média climatológica (1994 - 2018).

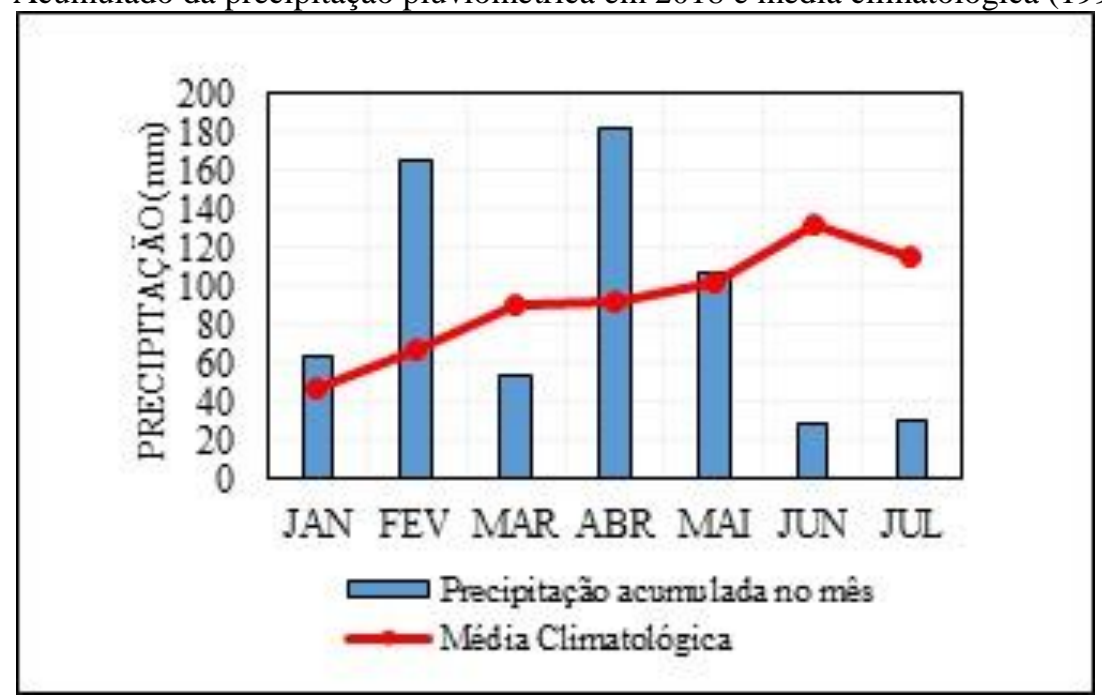

De janeiro até julho foram 91 dias com chuvas, ou seja 42,9\% do total de dias, sendo o mês de abril o que apresentou maior ocorrência de chuva, com 20 dias com chuva, acumulando um total de $182 \mathrm{~mm} /$ mensais, representando um aumento da pluviosidade de 
96,2\%, que foi bastante superior a média esperada para o mês, entretanto ressalta-se que não foi observado nenhum evento acima de $50 \mathrm{~mm} / \mathrm{dia}$.

Outro mês que chama atenção (Figura 3) é o mês de fevereiro que registrou um acumulo de $165 \mathrm{~mm} /$ mensais em apenas 13 dias com chuvas, aumento de 145,9\% do esperado para o mês. Neste mês, observou-se um evento de chuvas acima de $50 \mathrm{~mm} / \mathrm{dia}$ (chuva extrema) que ocorreu no dia 10 de fevereiro de 2018, com acumulado de 56,8 mm (Figura 4).

Segundo a reportagem no G1 Paraíba do dia 10 de fevereiro de 2018, o coordenador da Defesa Civil de Campina Grande, Ruiter Sansão, relatou que houve desabamentos de árvores, de muro de escola, alagamento em vias, queda de energia elétrica em alguns pontos da cidade, uma academia no bairro do Alto Branco ficou completamente alagada e famílias desabrigadas. A chuva intensa durou aproximadamente 2 hs e causou vários prejuizos na cidade.

Figura 4: Precipitação pluviométrica diária no mês de fevereiro de 2018.

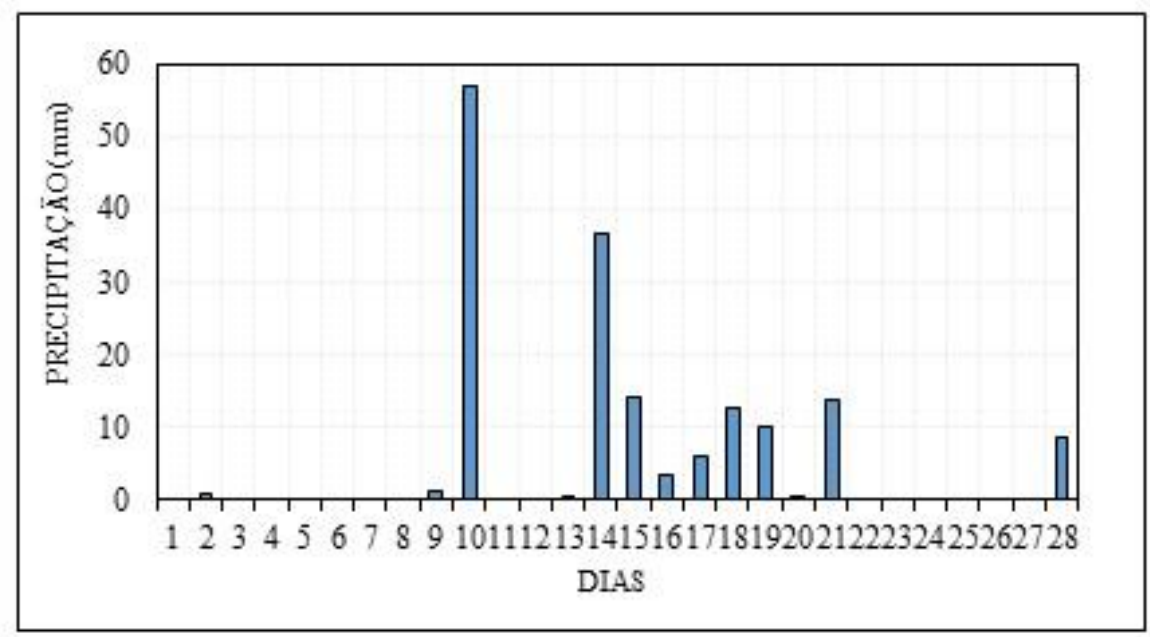

Conforme as informações do Boletim Técnico (CPTEC, 2018), o sistema atmosférico que influenciou as condições de tempo em boa parte da Região Nordeste, foi a presença de um Vórtice Ciclônico de Altos Níveis que combinada com a proximidade da Zona de Convergência Intertropical (ZCIT) favorece a formação da nebulosidade. Ressalta-se que são necessários estudos mais detalhados que analise não apenas o volume de chuvas diárias, como também chuvas que ocorram em dias consecutivos, tendo em vista que o acumulado de dias chuvosos também podem ocasionar danos a sociedade. 


\section{CONCLUSÕES}

Um total de 30 eventos de chuvas com intensidade acima de $50 \mathrm{~mm} / \mathrm{dia}$ foi identificado entre o período de 1994 a julho de 2018 na cidade de Campina Grande - PB. O ano de 2011 foi considerado a mais chuvoso da série, e também apresentou a maior quantidade de eventos extremos, 7 eventos. Eventos de chuvas com intensidade acima de 80 $\mathrm{mm} /$ dia foram apenas 5 eventos durante todo o período em análise.

Em escala mensal, os eventos extremos encontram-se dentro do período chuvoso do município, que compreende os meses de maio a julho.

No ano corrente, 2018, até o mês de julho foi observado apenas um evento no mês de fevereiro que ocorreu no dia 10 de fevereiro e que segundo a Defesa Civil causou vários prejuizos à sociedade de Campina Grande-PB.

\section{REFERENCIAS}

BRASIL. Ministério da Integração Nacional. Anuário brasileiro de desastres naturais: 2013.

Brasília: Ministério da Integração Nacional/Secretaria Nacional de Proteção e Defesa Civil/ Centro Nacional de Gerenciamento de Riscos e Desastres, 2014. Disponível em: http://www.mi.gov.br/c/document_library/get_file?uuid=fee4007a-ab0b-403e bb1a8aa00385630b\&groupId=10157. Acesso em: 22 fev. 2016.

CEPED/ UFSC. Universidade Federal de Santa Catarina. Centro Universitário de Estudos e Pesquisas sobre Desastres. Atlas brasileiro de desastres naturais 1991 a 2010: volume Brasil. Florianópolis: CEPED/UFSC, 2012.

DINIZ, J. M. T. Variabilidade da precipitação e do número de dias com chuvas de duas cidades distintas da Paraíba. HOLOS, Ano 29, vol 3, 2013.

GONÇALVES, N. M. S. Impactos pluviais e desorganização do espaço urbano em Salvador. p. 69-91. In: Clima Urbano. MONTEIRO, C. A. F; MENDONÇA, F. (Orgs.). São Paulo: Contexto, 2003.192p.

G1 Paraíba. Disponível em: <https://g1.globo.com/pb/paraiba/noticia/duas-horas-de-chuvaprovoca-alagamentos-e-desabamentos-em-campina-grande.ghtml>. Acesso em: 20 Agos. 2018.

LOUREIRO, Renata Silva de; et al. Estudo dos Eventos Extremos de Precipitação Ocorridos em 2009 no Estado do Pará. Revista Brasileira de Meteorologia, v. 29, n. esp., 83 - 94, 2014. 
MARENGO, J.A. et al., 2009: Future change of climate in South America in the late twentyfirst century: intercomparison of scenarios from three regional climate models. Climate Dynamics, 35(6), 1073- 1097, doi:10.1007/s00382-009-0721-6.

MINUZZI, R. B. SEDIYAMA, G. C. Influência da topografia na precipitação: uma análise estatística e via imagens de satélite. In. Congresso Brasileiro de Meteorologia, Fortaleza, 2004.

SANTOS, A.N.C. Influência da precipitação no rendimento agrícola do feijão (Phaseolus vulgaris L.) e algodão herbáceo (Gossypium hirsutum L.) em campina grande - pb. Dissertação - Curso em Meteorologia, Universidade Federal de Campina Grande, 2011.

SILVA, V.P.R; ALMEIDA, R.S.R. estudo do clima urbano na cidade de Campina Grande, PB. Rev. de Ci. Da vida, RJ, EDUR, v.32, n.1, jan/jun, p.31-44, 2012.

SILVA, N. T. Precipitações diárias intensas na cidade de João Pessoa, Paraíba. (Trabalho de Conclusão de Curso). Curso de Bacharelado em Geografia. João Pessoa: Departamento de Geociências - UFPB, 2014. 69p.

TEMOTEO, K.K.S. Eventos de chuvas intensas e extremas e seus impactos no espaço urbano de Campina Grande, Paraíba (Trabalho de Conclusão de Curso). Curso de Bacharelado em Geografia. João Pessoa: Departamento de Geociências - UFPB, 2016. 68p.

TEMOTEO, K.K.S; LIMA, E.R.R; MOURA, M. O. Eventos de chuvas intensas na cidade de Campina Grande, Paraíba. In: Variabilidade e Susceptibilidade Climática: Implicações Ecossistêmicas e Sociais. Anais...Goiânia: XII SBCG, 2016.

VIANELLO, R. L. Meteorologia básica e aplicações. $1^{\text {a }}$ Ed. Viçosa: Imprensa Universitária, 1991. 449 p.

ZANELLA, M. E; SALES, M. C. L.; ABREU, N. J. A análise das precipitações diárias intensas e impactos gerados em Fortaleza-CE. GEOUSP- Espaço e Tempo, São Paulo, ${ }^{\circ} 25$, p 53-68, 2009.

ZANELLA, M. E. Inundações em Curitiba: impactos, risco e vulnerabilidade socioambiental. 2. ed. Fortaleza: Editora da UFC, 2014. 197p.

Recebido em: 13/04/2019

Aceito para publicação em: 25/05/2019 